\title{
7.
}

\section{ON A CLASS OF DIFFERENTIAL EQUATIONS, AND ON THE LINES OF CURVATURE OF AN ELLIPSOID.}

[From the Cambridge Mathematical Journal, vol. III. (1843), pp. 264-267.]

CONSIDER the primitive equation

$$
f x+g y+h z+\ldots \ldots=0
$$

between $n$ variables $x, y, z$, the constants $f, g, h$ being connected by the equation

$$
H(f, g, h \ldots \ldots)=0
$$

$H$ denoting a homogeneous function. Suppose that $f, g, h \ldots \ldots$ are determined by the conditions

$$
\begin{array}{ccc}
f x_{1}+g y_{1}+h z_{1} & \ldots \ldots=0 \\
\vdots & \vdots & \vdots \\
f x_{n-2}+g y_{n-2} & +h z_{n-2} \ldots \ldots= & \\
&
\end{array}
$$

$$
X=\left|\begin{array}{ccc}
y & , z & , \ldots \\
y_{1} & , z_{1} & , \ldots \\
\vdots & \vdots & \\
y_{n-2}, & z_{n-2}, \ldots
\end{array}\right|
$$

Then writing

; the equations (3)

(3) give $f, g, h$

proportional to $x, y, z, \ldots \ldots$ or eliminating $f, g, h \ldots \ldots$ by the equation (2),

$$
H(X, Y, Z \ldots \ldots)=0 \text {. }
$$

Conversely the equation (5), which contains, in appearance, $n(n-2)$ arbitrary constants, is equivalent to the system (1), (2). And if $H$ be a rational integral function of the order $r$, the first side of the equation (5) is the product of $r$ factors, each of them of the form given by the system (1), (2). 
Now from the equation (1), we have the system

$$
\begin{array}{cccc}
f x+g y+h z & \ldots \ldots=0 \\
f d x+g d y+h d x & \ldots \ldots=0 \\
\vdots & \vdots & \vdots & \\
f d^{n-2} x+g d^{n-2} y+h d^{n-2} z & \ldots \ldots= & \\
& &
\end{array}
$$

or writing

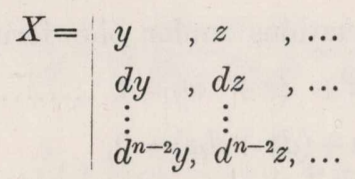

with analogous expressions for $Y, Z \ldots \ldots$; then from the equations $(6), f, g, h \ldots \ldots$ are proportional to $X, Y, Z \ldots \ldots$ : or, eliminating by (2),

$$
H(X, Y, Z \ldots \ldots)=0 \text {. }
$$

Conversely the integral of the equation (8) of the order $(n-2)$ is given either by the system of equations (1), (2), in which it is evident that the number of arbitrary constants is reduced to $(n-2)$; or, by the equation (5), which contains in appearance $n(n-2)$ arbitrary constants, but which we have seen is equivalent in reality to the system (1), (2).

Thus, with three variables, the integral of

$$
H(y d z-z d y, z d x-x d z, x d y-y d x)=0
$$

may be expressed in the form

$$
H\left(y z_{1}-y_{1} z, \quad z x_{1}-z_{1} x, \quad x y_{1}-x_{1} y\right)=0
$$

and also in the form

$$
\begin{array}{r}
f x+g y+h z=0 \\
H(f, g, h)=0
\end{array}
$$

The above principles afford an elegant mode of integrating the differential equation for the lines of curvature of an ellipsoid. The equation in question is

$$
\left(b^{2}-c^{2}\right) x d y d z+\left(c^{2}-a^{2}\right) y d z d x+\left(a^{2}-b^{2}\right) z d x d y=0 .
$$

where $x, y, z$ are connected by

$$
\frac{x^{2}}{a^{2}}+\frac{y^{2}}{b^{2}}+\frac{z^{2}}{c^{2}}=1
$$

writing

$$
\frac{x^{2}}{a^{2}}=u, \quad \frac{y^{2}}{b^{2}}=v, \quad \frac{z^{2}}{c^{2}}=w
$$

these become

$$
\begin{array}{r}
\left(b^{2}-c^{2}\right) u d v d w+\left(c^{2}-a^{2}\right) v d w d u+\left(a^{2}-b^{2}\right) w d u d v=0 . \\
u+v+w=1 \ldots \ldots \ldots \ldots \ldots \ldots \ldots \ldots \ldots \ldots \ldots
\end{array}
$$


Multiplying by

$$
-\{(v d u-u d v)(w d v-v d w)(u d w-w d u)\}^{-1}
$$

the first of these becomes

$\frac{-a^{2} d u}{(v d u-u d v)(u d w-w d u)}+\frac{-b^{2} d v}{(w d v-v d w)(v d u-u d v)}+\frac{-c^{2} d w}{(u d w-w d u)(w d v-v d w)}=0 \ldots$

but writing (17) and its derived equations under the form

$$
\begin{array}{r}
u+(v+w)=1 \\
d u+(d v+d w)=0
\end{array}
$$

we deduce

$$
-d u(v+w)+u(d v+d w)=-d u
$$

i.e.

$-d u=-(v d u-u d v)+(u d w-w d u)$

and similarly

$$
\begin{aligned}
& -d v=-(w d v-v d w)+(v d u-u d v) \\
& -d w=-(u d w-w d u)+w d v-v d w)
\end{aligned}
$$

Substituting,

$$
\frac{b^{2}-c^{2}}{w d v-v d w}+\frac{c^{2}-a^{2}}{u d w-w d u}+\frac{a^{2}-b^{2}}{v d u-u d v}=0
$$

the integral of which may be written in the form

$$
\frac{b^{2}-c^{2}}{w v_{1}-v w_{1}}+\frac{c^{2}-a^{2}}{u w_{1}-w u_{1}}+\frac{a^{2}-b^{2}}{v u_{1}-v_{1} u}=0
$$

where, on account of (17),

$$
u_{1}+v_{1}+w_{1}=1
$$

and also in the form

$$
f u+g v+h w=0
$$

where $f, g, h$ are connected by

$$
\frac{b^{2}-c^{2}}{f}+\frac{c^{2}-a^{2}}{g}+\frac{a^{2}-b^{2}}{g}=0
$$

this last equation is satisfied identically by

$$
f=\frac{b^{2}-c^{2}}{B^{2}-C^{2}}, \quad g=\frac{c^{2}-a^{2}}{C^{2}-A^{2}}, \quad h=\frac{a^{2}-b^{2}}{A^{2}-B^{2}}
$$

Restoring $x, y, z, x_{1}, y_{1}, z_{1}$ for $u, v, w, u_{1}, v_{1}, w_{1}$, the equations to a line of curvature passing through a given point $x_{1}, y_{1}, z_{1}$, on the ellipsoid, are the equation (14) and

$$
\frac{\left(b^{2}-c^{2}\right)}{a^{2}\left(y_{1}^{2} z^{2}-y^{2} z_{1}^{2}\right)}+\frac{\left(c^{2}-a^{2}\right)}{b^{2}\left(z_{1}^{2} x^{2}-z^{2} x_{1}^{2}\right)}+\frac{\left(a^{2}-b^{2}\right)}{c^{2}\left(x_{1}^{2} y^{2}-x^{2} y_{1}^{2}\right)}=0
$$

or again, under a known form, they are the equation.(14) and

$$
\frac{\left(b^{2}-c^{2}\right)}{B^{2}-C^{2}} \frac{x^{2}}{a^{2}}+\frac{c^{2}-a^{2}}{C^{2}-A^{2}} \frac{y^{2}}{b^{2}}+\frac{a^{2}-b^{2}}{A^{2}-B^{2}} \frac{z^{2}}{c^{2}}=0
$$


From the equations (14), (29) it is easy to prove the well-known form

$$
\frac{x^{2}}{a^{2}+\theta}+\frac{y^{2}}{b^{2}+\theta}+\frac{z^{2}}{c^{2}+\theta}=1 \text {. }
$$

in fact, multiplying (29) by $m$, and adding to (14), we have the equation (30), if the equations

$$
\begin{aligned}
& \frac{1}{a^{2}}+m \frac{b^{2}-c^{2}}{B^{2}-C^{2}} \frac{1}{a^{2}}=\frac{1}{a^{2}+\theta}, \\
& \frac{1}{b^{2}}+m \frac{c^{2}-a^{2}}{C^{2}-A^{2}} \frac{1}{b^{2}}=\frac{1}{b^{2}+\theta}, \\
& \frac{1}{c^{2}}+m \frac{a^{2}-b^{2}}{A^{2}-B^{2}} \frac{1}{c^{2}}=\frac{1}{\left(c^{2}+\theta\right)},
\end{aligned}
$$

are satisfied.

But on reduction, these take the form

$$
\begin{aligned}
& \left(B^{2}-C^{2}\right) \theta+\left(b^{2}-c^{2}\right) m \theta+m a^{2}\left(b^{2}-c^{2}\right)=0, \\
& \left(C^{2}-A^{2}\right) \theta+\left(c^{2}-a^{2}\right) m \theta+m b^{2}\left(c^{2}-a^{2}\right)=0, \\
& \left(A^{2}-B^{2}\right) \theta+\left(a^{2}-b^{2}\right) m \theta+m c^{2}\left(a^{2}-b^{2}\right)=0,
\end{aligned}
$$

and since, by adding, an identical equation is obtained, $m$ and $\theta$ may be determined to satisfy these equations. The values of $\theta, m$ are

$$
\begin{aligned}
& \theta=\frac{\left(a^{2}-b^{2}\right)\left(b^{2}-c^{2}\right)\left(c^{2}-a^{2}\right)}{a^{2}\left(B^{2}-C^{2}\right)+b^{2}\left(C^{2}-A^{2}\right)+c^{2}\left(A^{2}-B^{2}\right)} \cdots \cdots \\
& m=\frac{b^{2} c^{2}\left(B^{2}-C^{2}\right)+c^{2} a^{2}\left(C^{2}-A^{2}\right)+a^{2} b^{2}\left(A^{2}-B^{2}\right)}{\left(a^{2}-b^{2}\right)\left(b^{2}-c^{2}\right)\left(c^{2}-a^{2}\right)}
\end{aligned}
$$

